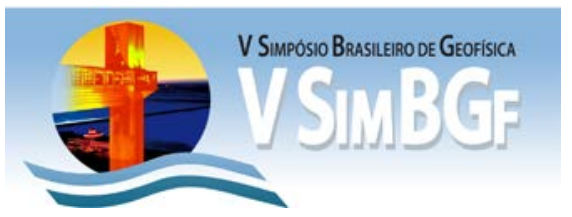

\title{
Isótopos Estáveis em sedimentos marinhos e águas da Baía de Todos os Santos: Indicadores de Fontes e Preservação
}

\author{
José Roberto Bispo de Souza*1; Maria do Rosário Zucchi ${ }^{1}$; Alexandre Barreto Costa ${ }^{1}$; Antônio Expedito Gomes de Azevedo ${ }^{1}$ \\ ${ }^{1}$ CPGG-IF-Universidade Federal da Bahia (UFBA)
}

Copyright 2012, SBGf - Sociedade Brasileira de Geofísica

Este texto foi preparado para a apresentação no V Simpósio Brasileiro de Geofísica, Salvador, 27a 29 de novembro de 2012. Seu conteúdo foi revisado pelo Comitê Técnico do VSimBGf, mas não necessariamente representa a opinião da SBGf ou de seus associados. É proibida a reprodução total ou parcial deste material para propósitos comerciais sem prévia autorização da SBGf.

\section{Resumo}

In this work we use the $\delta^{13} \mathrm{C}$ and $\delta^{15} \mathrm{~N}$ of surficial sediments and physico-chemical parameters of the water sampled in 14 points of the Todos os Santos Bay to analyze the origin of the organic matter in the sediments. It was characterized the region influenced by input from the Subaé and Paraguaçu rivers with the larger values of $\delta^{13} C_{D I C}$ in the sediment and dissolved inorganic carbon of water, and larger values of $\mathrm{TOC}$ and $\mathrm{C} / \mathrm{N}$ ratio. The isotopic ratio of carbon and nitrogen indicates a significant impact of pollution by sewage and terrestrial material in the sediments of the Bay. Results were analyzed using multivariate statistics (PCA) analysis showing two sample group referring to the west and east BTS.

\section{Introdução}

A poluição marinha tem sido alvo de grande atenção da comunidade científica nas últimas décadas. Durante séculos, o homem tem despejado detritos de origens diversas nos oceanos indiscriminadamente. Além disso, os oceanos também têm sido usados como fonte de alimentos, recursos minerais e para recreação, sem preocupação com o impacto que esta poluição possa estar causando ao ambiente marinho. Devido à vastidão dos oceanos, estes efeitos ainda não são evidentes, mas a acumulação e o aumento desses impactos já tem resultado em alterações de alguns sistemas marinhos, merecendo cuidados cada vez maiores. Com o aumento populacional e a concentração de pólos industriais na zona costeira, os impactos antrópicos nestes locais tendem a aumentar, afetando principalmente um dos tipos de ecossistemas mais produtivos do planeta, os estuários e, consequentemente, ao homem.

Da metade do século $X X$ até os dias atuais, a baía de Todos os Santos vem sofrendo uma forte influência antrópica devida ao processo desordenado de ocupação de seu entorno e do tráfego e exploração de petróleo. Nas redondezas da baía são encontrados os mais elevados índices de densidade demográfica do Estado. Nos dezesseis municípios que são banhados por suas águas, vivem cerca de três milhões de habitantes juntamente com um parque industrial fundamentado na indústria química e de exploração, refino e processamento de petróleo. Boa parte dos esgotos urbanos é lançada diretamente na baía sem qualquer tratamento. Os efluentes líquidos e emissões atmosféricas das indústrias ainda são monitorados de forma precária. Da mesma forma, os resíduos sólidos urbanos e industriais ainda não encontram disposição mal adequada.

Neste estudo, os isótopos estáveis foram usados como traçadores ambientais. A caracterização isotópica da matéria orgânica presente nos sedimentos permitiu avaliar fontes antrópicas que contribuíram com o material orgânico encontrado. As técnicas isotópicas em conjunto com os parâmetros físico-químico constituem uma poderosa ferramenta na caracterização ambiental.

\section{Metodologia/ Problema Investigado}

A área de estudo foi baía de Todos os Santos (Figura 1). Ela é uma das maiores baías do Brasil com $1.086 \mathrm{~km}^{2}$ de extensão e profundidade média de $6 \mathrm{~m}$, atingindo profundidades de até $102 \mathrm{~m}$. Possui uma rara beleza e potencial turístico, abrigando os manguezais dos estuários dos rios Paraguaçu, Subaé, Jaguaripe, Cobre, dentre outros, em mais de $60 \%$ de seu perímetro. O clima da baía de Todos os Santos é classificado como quente e úmido, com pluviosidade média de $1.800 \mathrm{~mm} . \mathrm{a}^{-1}$, sendo que a maioria das precipitações concentra-se de abril a julho. Em seu interior, os ventos são geralmente de nordeste no verão e de sudeste no inverno. A amplitude máxima das marés é de 3,60 m. A circulação de águas na baía é conduzida primariamente por marés, as quais são do tipo semidiurno, com duas baixa-mares e duas preamares em um período de, aproximadamente, 24,5 $h$.

Os pontos amostrados estão ilustrados na Figura 1. Foram coletadas em cada ponto três amostras de água superficial e sedimento de fundo com o objetivo de observar a variabilidade local e a variabilidade total na região estudada. As amostras sofreram os mesmos tratamentos em cada etapa do trabalho.

As amostras de sedimento de fundo, coletadas com o auxílio de uma draga do tipo Van Veen, foram condicionadas em recipiente de vidro previamente descontaminados e mantidas refrigeradas até a chegada ao laboratório. No laboratório as amostras foram congeladas e mantidas à temperatura em torno de $-20^{\circ} \mathrm{C}$ até sua liofilização. Após a liofilização as amostras foram moídas a pó fino, aproximadamente 140 mesh.

As amostras de água coletadas em superfície e condicionadas em recipientes de vidro de $100 \mathrm{ml}$ previamente descontaminados, fixadas com $\mathrm{HgCl}_{2}$ (cloreto de mercúrio) e mantidas refrigeradas até a 
chegada ao laboratório e mantidas nesta condição até a realização das analises. Os parâmetros físico-químicos da água, pH, salinidade, oxigênio dissolvido, medidos no local (in situ) com o auxílio de uma sonda Hanna Hi 9828.

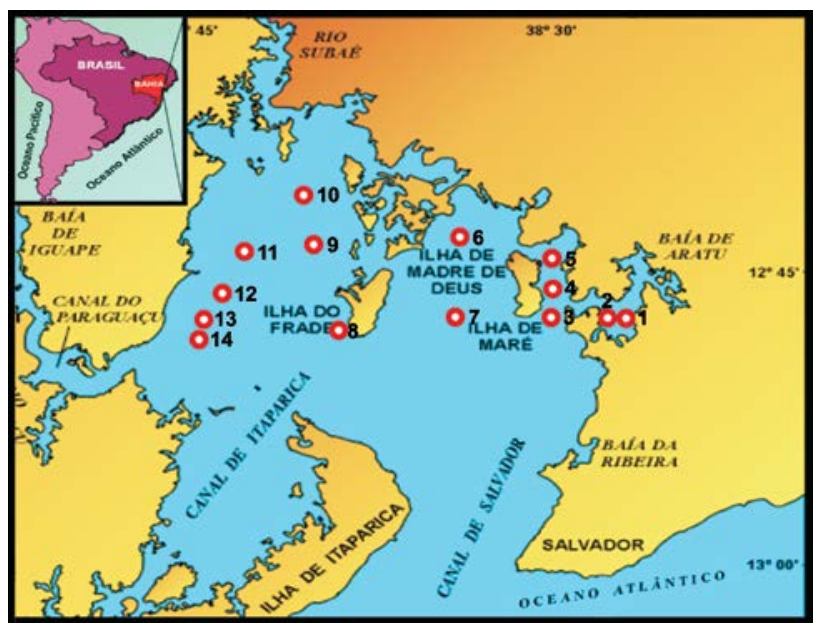

Figura 1. Localização das amostragens na BTS (modificado de Lessa et. al., 2000)

Para a análise dos sedimentos, foi feito ataque ácido com $10 \mathrm{~mL}$ de ácido clorídrico $(\mathrm{HCl}) 1 \mathrm{~mol}^{-1} \mathrm{~L}^{-1}$ em $0,5 \mathrm{~g}$ de cada amostra para retirar o carbonato. Após 24h, as amostras foram centrifugadas para extrair o líquido sobrenadante, depois lavadas com água deionizada e centrifugadas por três vezes e, finalmente seca a temperaturas entre 35$50^{\circ} \mathrm{C}$. Depois do tratamento, cerca de $8 \mathrm{mg}$ de cada amostra seca foi pesada numa cápsula de estanho para fazer a análise elementar da quantidade e da razão isotópica de Carbono Orgânico e nitrogênio, utilizando um analisador elementar Costech acoplado a um espectrômetro de massa ThermoFinnigan Delta Plus. Os valores isotópicos foram referenciados em relação a duas referências certificadas pela Agência Internacional de Energia Atômica (AIEA) (USGS40 e USGS41).

Para a análise de do carbono inorgânico dissolvido, foram colocados $700 \mu \mathrm{L}$ em um recipiente com $30 \mu \mathrm{L}$ de ácido fosfórico pressurizado com gás hélio, onde é liberado o gás carbônico. Após um tempo de equilíbrio de 18 horas numa temperatura de $25^{\circ} \mathrm{C}$, e analisados em um espectrômetro de massa ThermoFinnigan Delta Plus acoplado ao módulo de preparação Gas bench. Os carbonatos de sódio e de lítio, utilizados como padrões secundários, foram aferidos com o padrão NBS 19 da AIEA.

Os resultados das análises foram reportados em relação ao PDB (Carbono) e ao Ar atmosférico (Nitrogênio), sendo:

$$
\delta^{\mathrm{y}} \mathrm{X}=\left(\frac{\mathrm{R}_{\text {amostra }}-\mathrm{R}_{\text {padrão }}}{\mathrm{R}_{\text {padrão }}}\right) \times 100 \%
$$

onde ${ }^{\mathrm{y}} \mathrm{X}={ }^{13} \mathrm{C}$ (carbono) ou ${ }^{15} \mathrm{~N}$ (nitrogênio) e $\mathrm{R}={ }^{13} \mathrm{C} /{ }^{12} \mathrm{C}$ para carbono ou $R={ }^{15} \mathrm{~N} /{ }^{14} \mathrm{~N}$ para o nitrogênio.
Na tabela 1 e 2 a seguir estão ilustrados os valores médios, máximos, mínimos e o desvio padrão das variáveis analisadas.

Tabela 1. Valores médios, máximos, mínimos e desvio padrão dos parâmetros físico-quimicos.

\begin{tabular}{|c|c|c|c|c|c|c|}
\cline { 2 - 7 } \multicolumn{1}{c|}{} & $T$ & $p H$ & ORP & OD \% & $m$ S/cm & Salin. \\
\hline média & 25,18 & 8,22 & -14 & 8,0 & 52,2 & 34,3 \\
\hline max & 25,53 & 8,33 & 72 & 25,1 & 53,5 & 35,4 \\
\hline $\min$ & 24,65 & 8,06 & -82 & 1,0 & 50,1 & 32,8 \\
\hline$D P$ & 0,26 & 0,07 & 42 & 6,4 & 1,5 & 1,1 \\
\hline
\end{tabular}

Tabela 2. Valores médios, máximos, mínimos e desvio padrão dos parâmetros atômicos (massa) e isotópicos.

\begin{tabular}{|c|c|c|c|c|c|c|}
\cline { 2 - 7 } \multicolumn{1}{c|}{} & $C O T$ & $N$ & $C / N$ & $\delta^{13} C$ & $\delta^{13} N$ & $\delta^{13} C_{D I C}$ \\
\hline media & 1,21 & 0,20 & 6,2 & $-21,4$ & 5,1 & $-0,41$ \\
\hline $\max$ & 1,64 & 0,28 & 8,7 & $-19,5$ & 6,1 & 0,19 \\
\hline $\min$ & 0,71 & 0,13 & 5,3 & $-22,8$ & 4,1 & $-1,15$ \\
\hline$D P$ & 0,25 & 0,04 & 1,0 & 1,0 & 0,7 & 0,42 \\
\hline
\end{tabular}

Para entender as correlações entre os isótopos ambientais e as variáveis físico-químicas, utilizamos técnicas estatísticas da análise de componente principais (PCA). A visualização das diversas variáveis nas amostras de sedimento e água de cada ponto amostrado foi possível ser analisada e assim identificar a relação entre as características dos dados e seus possíveis agrupamentos.

Em uma análise de componentes principais, define-se a estrutura dos dados através de gráficos de scores e loadings cujos eixos são componentes principais (CPs) nos quais os dados são projetados. Os scores fornecem a projeção dos dados no espaço das CPs, enquanto os loadings fornecem a significância relativa em relação às variáveis originais. Como as CPs são ortogonais, é possível examinar as relações entre amostras e variáveis através dos gráficos dos scores e dos loadings. O estudo do conjunto de scores e loadings ainda permite estimar a influência de cada variável em cada amostra.

A Figura 2a ilustra o PC1 versus PC2 (loadings) dos parâmetros medidos da água. A salinidade, temperatura, o oxigênio dissolvido e o $\delta^{13} C_{D I C}$ são os parâmetros que tem maiores valores de PC1, enquanto o PC2 apresentou maiores valores da variável oxigênio dissolvido.

Nos gráficos do PC1 versus PC2 as amostras estão grupadas pela semelhança de suas variáveis, especialmente pelo teor dos componentes principais. Através da PCA verificou-se que com as duas primeiras componentes principais é possível descrever $84,7 \%$ nos valores, sendo $57,3 \%$, da variância total descrita pela primeira componente principal (CP1) e, 27,4\% pela CP2.

\section{Resultados}



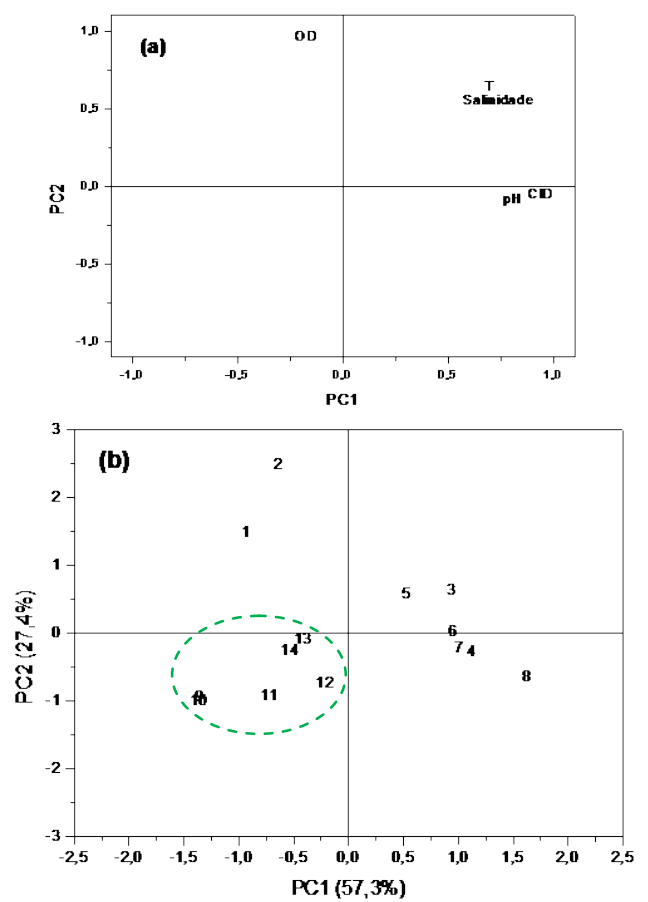

Figura 2. (a) Gráfico de PC1 versus PC2, das variáveis; (b) Gráfico de PC1 versus PC2, das amostras de água.

A Figura $2 \mathrm{~b}$ ilustra o gráfico da $\mathrm{PC} 1$ versus $\mathrm{PC} 2$, onde evidencia-se que os pontos 1 e 2 (Baía de Aratu) apresentam maiores valores do parâmetro oxigênio dissolvido. Os pontos 3 (Canal de Aratu), 4 (Porto de Aratu), 5 (Caboto), 6 (RELAM), 7 (Canal de Madre de Deus) e 8 (Ponte de Nossa Senhora) apresentam altos valores dos parâmetros salinidade, temperatura, $\mathrm{pH}$ e $\delta^{13} C_{C I D}$ sendo esses pontos estão localizados no lado leste da Baía, em mar aberto. Os pontos 9 (Ilha das Vacas), 10 (Bimbarra), 11 (Saubara), 12, 13 e 14 (Bom Jesus dos Pobres) apresentam comportamento semelhantes e valores mais baixos dos parâmetros salinidade, temperatura, $\mathrm{pH}$ e $\delta^{13} \mathrm{C}_{\mathrm{CID}}$. Estes pontos estão relacionados com a parte oeste da Baía de Todos os Santos, próximos da desembocadura dos rios Subaé e Paraguaçu. Observa-se também um gradiente decrescente em PC1 e crescente em PC2 formados pelos pontos 8, 4, 7, 6, 5, 3, 2 e 1 .

A Figura 3a ilustra o PC1 versus PC2 (loadings) dos parâmetros medidos para do sedimento. $\mathrm{O} \delta^{13} \mathrm{C}$ e o carbonato apresentam valores positivos de PC1. O PC2 apresentou valores positivos de $\delta^{15} \mathrm{~N}$, COT e N.

Nos gráficos do PC1 versus PC2 as amostras estão grupadas pela semelhança de suas variáveis, onde verificou-se que com as duas primeiras componentes principais é possível descrever $79,9 \%$ nos valores, sendo $48,5 \%$, da variância total descrita pela primeira componente principal (CP1) e, 31,4\% pela CP2.

A Figura 3b ilustra o gráfico da PC1 versus PC2 das amostras de sedimento onde, mais uma vez, é evidenciada a diferença de comportamento dos pontos 9 , $10,11,12,13$ e 14, localizados na parte oeste da BTS, concentrados em um quadrante (PC1 negativo e PC2 positivo) com relação ao demais. Estas amostras apresentam altos teores de sílica de $\mathrm{N}$ e COT, sofrem o efeito da desembocadura dos rios Subaé e Paraguaçu. Os pontos 1, 2 e 5 (PC1 negativo e PC2 negativo) e os pontos 3 e 4 (PC1 positivo e PC2 negativo), apresentam mais baixos valores desses parâmetros, enquanto que os pontos 6 e 7 apresentam comportamento semelhante e maiores valores de carbonato, $\delta^{13} \mathrm{C}, \mathrm{N}$ e $\delta^{15} \mathrm{~N}$.
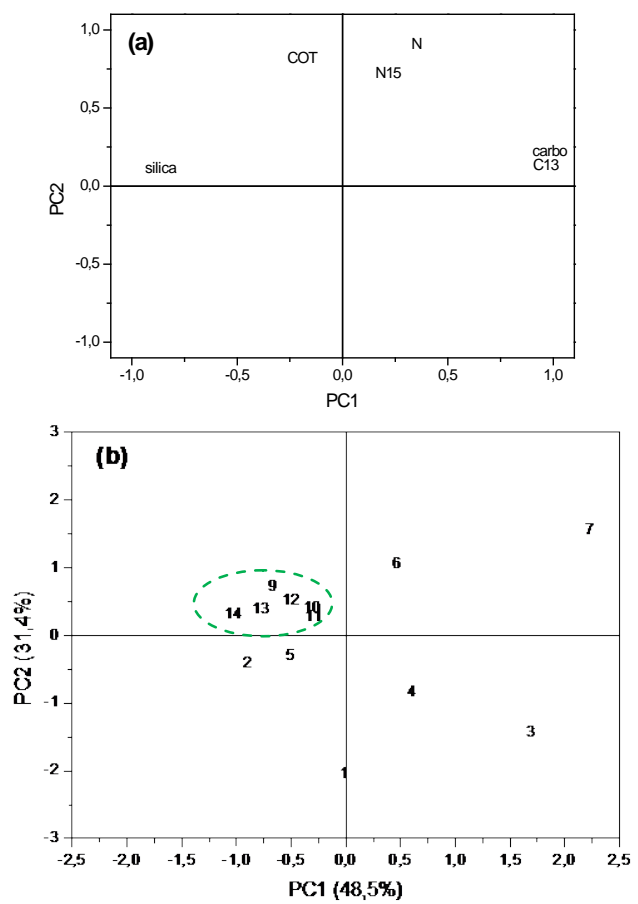

Figura 3. (a) Gráfico de PC1 versus PC2, das variáveis; (b) Gráfico de PC1 versus PC2, das amostras de sedimento de fundo.

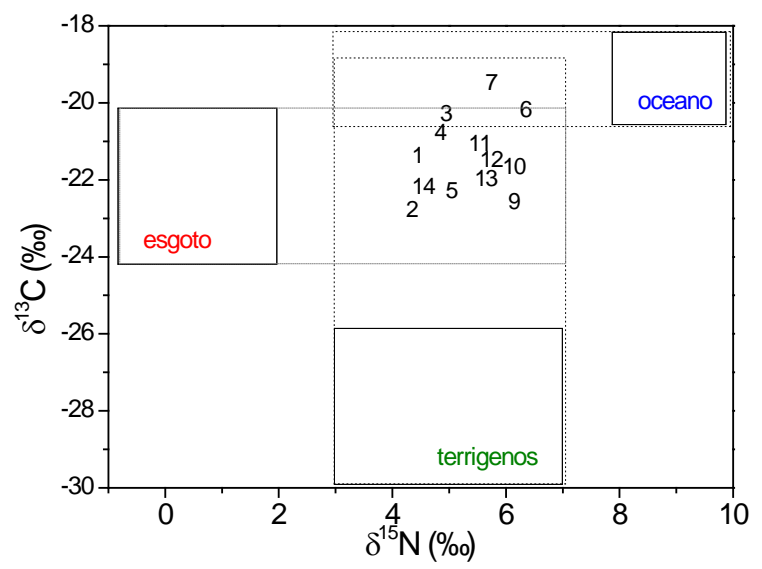

Figura 4. Grafico $\delta^{13} \mathrm{C}$ versus $\delta^{15} \mathrm{~N}$ para o sedimento de fundo na BTS

Denies (1980) fez uma classificação para os valores isotópicos do $\mathrm{C}$ e $\mathrm{N}$ referentes a origem da matéria orgânica total em sedimentos. Baseado nesta classificação (Figura 4), os valores encontrados estão ilustrados indicando uma mistura entre os componentes. 
Em relação ao $\delta^{13} \mathrm{C}$ os valores variam entre esgoto $\mathrm{e}$ marinho e em relação ao $\delta^{15} \mathrm{~N}$ os valores obtidos estão na faixa dos terrígenos (Barros, 2010; Sampaio, 2010). Os valores isotópicos do $\mathrm{N}$ e do $\mathrm{C}$ indicam, para a maioria das amostras, influência de material orgânico oriundo de esgoto e material terrígeno.

\section{Discussão e Conclusões}

Os resultados obtidos mostram que os pontos 1 e 2 (Baía de Aratu), apresentam valores mais altos do parâmetro: oxigênio dissolvido. Os pontos 3 (Canal de Aratu), 4 (Porto de Aratu), 5 (Caboto), 6 (RELAM), 7 (Canal de Madre de Deus) e 8 (Ponte de Nossa Senhora) apresentam altos valores dos parâmetros salinidade, temperatura, $\mathrm{pH}$ e $\delta^{13} \mathrm{C}_{\mathrm{CID}}$ sendo esses pontos estão localizados no lado leste da Baía, referente a parte da Baía mais aberta ao mar. Os pontos 9 (Ilha das Vacas), 10 (Bimbarra), 11 (Saubara), 12, 13 e 14 (Bom Jesus dos Pobres) apresentam comportamento similar apresentando valores mais baixos de $\mathrm{pH}$, salinidade, OD e temperatura e valores mais altos de COT, C/N. Estes pontos estão localizados na parte oeste da Baía de Todos os Santos, próximos da desembocadura dos rios Subaé e Paraguaçu.

As análises isotópicas do $\mathrm{N}$ e $\mathrm{C}$ nas amostras de sedimento indicam que, para a maioria das amostras, ocorre a influência de material orgânico oriundo de esgoto e material terrígeno, mostrando que o material depositado na baía sofre grande impacto da poluição.

\section{Agradecimentos}

FAPESB pelo apoio financeiro e aos oceanógrafos Yuri Costa e Antônio Dórea pelo auxílio na amostragem.

\section{Referências}

Barros G.V.,Martinelli L.A., Oliveira Novais T.M., Ometto J.P., Zuppi G.M. 2010. Stable isotopes of bulk organic matter to trace carbon and nitrogen dynamics in an estuarine ecosystem in Babitonga Bay (Santa Catarina, Brazil).Science of The Total Environment 408: 22262232.

Deines P. 1980.The isotopic composition of reduced organic carbon.In: P. Fritz \&J. Ch. Fontes (eds.), Handbook of Environmental Isotope Geochemistry. Volume 1: The Terrestrial Environment, Elsevier Science Ltd, p.: 329-406.

Lessa G., Lima G., Carvalho J. e Oliveira W. (2000) Oceanografia Física e Geológica da Baía de Todos os Santos., http://www.cpgg.ufba.br/glessa/bts.

Sampaio L., Freitas R., Máguas C., Rodrigues A., Quintino V. 2010. Coastal sediments under the influence of multiple organic enrichment sources: An evaluation using carbon and nitrogen stable isotopes. Marine Pollution Bulletin, 60: 272-282. 